\title{
Taxonomie de l'approche par compétences intégrée au regard de la complexité. Contribution critique à la santé publique
}

Adapting our planning tools to expected epistemological orientations:

introducing taxonomy associated with the integrated competency-based model

in health education.(Essay of) Critical contribution to public health

Florence Parent, Jean-Marie De Ketele, Fabienne Gooset et Manoé Reynaerts

\section{OpenEdition}

Journals

Édition électronique

URL : https://journals.openedition.org/trema/5907

DOI : $10.4000 /$ trema. 5907

ISSN : 2107-0997

Éditeur

Faculté d'Éducation de l'université de Montpellier

Référence électronique

Florence Parent, Jean-Marie De Ketele, Fabienne Gooset et Manoé Reynaerts, « Taxonomie de l'approche par compétences intégrée au regard de la complexité. Contribution critique à la santé publique », Tréma [En ligne], 54 | 2020, mis en ligne le 01 décembre 2020, consulté le 28 juin 2022 URL : http://journals.openedition.org/trema/5907 ; DOI : https://doi.org/10.4000/trema.5907

Ce document a été généré automatiquement le 14 décembre 2020.

Trema 


\title{
Taxonomie de l'approche par compétences intégrée au regard de la complexité. Contribution critique à la santé publique
}

\author{
Adapting our planning tools to expected epistemological orientations: \\ introducing taxonomy associated with the integrated competency-based model \\ in health education.(Essay of) Critical contribution to public health
}

Florence Parent, Jean-Marie De Ketele, Fabienne Gooset et Manoé Reynaerts

Nous remercions Gregory Aiguier, Isabelle François, Jean Jouquan, Colette Lejeune et Helyett Wardavoir (enseignante-chercheure, département santé, HELB-IP) pour leurs contributions lors de la relecture du manuscrit.

\section{Introduction}

« Nietzsche sortant d'un hôtel de Turin. Il aperçoit devant lui un cheval et un cocher qui le frappe à coups de fouet. Nietzsche s'approche du cheval, il lui prend l'encolure entre les bras sous les yeux du cocher et il éclate en sanglots. (...) C'est précisément à ce moment-là que s'est déclarée sa maladie mentale. Mais selon moi, c'est bien là ce qui donne à son geste sa profonde signification. Nietzsche était venu demander au cheval pardon pour Descartes. Sa folie (donc son divorce avec l'humanité) commence à l'instant où il pleure sur le cheval »

Milan Kundera, L'insoutenable légèreté de l'être. Gallimard, Paris, 1884

\section{Contexte, problématique et postulats}

Dans la perspective d'une optimisation des pratiques professionnelles au regard des besoins des systèmes de santé, l'élaboration d'un curriculum de formation 
professionnelle initiale ou continue en santé participe d'une démarche de planification. Notre option est de considérer d'une part, le contexte de la formation comme un lieu de planification quels que soient les vocables utilisés (ingénierie; programmation; régulation; objectifs; acquis d'apprentissage etc.) et de l'autre, que le secteur de la santé devrait s'intéresser de manière critique à ces planifications dans une visée d'intersectorialité (santé et éducation) responsable. En effet, lorsque l'on envisage des activités d'enseignement et d'apprentissage avec des résultats attendus formulés en termes de compétences, il est essentiel de s'adosser à un construit qui rende compte le plus adéquatement possible des unes et des autres. C'est précisément le rôle d'une taxonomie. Définie comme étant une "classification systématique et hiérarchisée d'objectifs d'habileté, indépendante des objectifs de contenu, définis avec précision et agencés selon un continuum de complexité croissante de développement [...]» (Legendre, 2006) son rôle est de structurer les finalités d'un programme éducatif, de l'évaluer et de le réguler (Parent \& Jouquan, 2014).

2 La problématique liée à ces cadres théoriques est, d'une part, leur «invisible présence », et, de l'autre, leur impact.

3 Par «invisible présence ", nous entendons le manque, voire l'absence, de débats critiques et ouverts au sein des institutions de formation en santé quant aux orientations épistémologiques que de tels outils de planification engendrent nécessairement.

4 Par impact, nous entendons la même faille relevée cette fois au sein du système de santé quant aux liens entre l'épistémologie qui détermine nos modes de penser et d'agir et la qualité des pratiques professionnelles et de l'offre de services de santé attendus.

5 Or, la formation des soignants, particulièrement celle des médecins, reste fondamentalement structurée à partir d'une logique binaire, théorie et pratique, amenant à la construction de curriculums qui, en suivant cette logique formelle, perdent leur pertinence et leur efficience comme nous l'argumentons au fil de nos développements.

6 En effet, de telles orientations sont favorisées par l'usage de taxonomies souvent implicitement à l'œuvre et qui peuvent être tenues pour réductrices.

7 Ainsi, nous centrons la présente contribution sur le débat de la complexité en éducation, dans un contexte spécifique celui des planifications curriculaires et des programmes de formation en santé, et sur un objet particulier, celui des taxonomies en éducation.

Pour cela nous partons d'un triple postulat :

- Epistémologique : celui d'un glissement de l'objet d'enseignement et d'apprentissage du savoir vers la pratique considérant l'apprentissage comme une activité sociocognitive et la médecine comme étant avant tout une pratique, accompagnée de sciences et instrumentée par des techniques (Folscheid, 1996). Ce premier postulat se fonde sur la perspective des épistémologies constructivistes telles qu'elles ont été explicitées par Jean-Louis Le Moigne (Le Moigne, 2012) ou, plus récemment, par Pierre Steiner argumentant la nécessité épistémologique d'une « centralité de l'action » (Steiner, 2008);

- Anthropologique: celui d'une ouverture à la pluralité des savoirs (Coutellec, 2015; Schurmans, 2006) permettant de rencontrer l'être humain dans toute sa complexité en référence à l'attitude d'ouverture dialogique de Morin (Morin, 1990). Ce second postulat se 
fonde sur une perspective anthropologique de la personne et des communautés visant à considérer autant les dimensions de santé que celles des vécus et des inscriptions sociales et contextuelles et du Sujet et de la santé ;

- Ethique : celui d'une interprétation non téléologique de l'intentionnalité de l'agir humain en référence aux travaux de Hans Joas (Joas, 2001), permettant de développer une perspective originale quant aux aspects éthiques ou axiologiques qui se penchent sur le sens, la valeur de l'activité et de l'action en tant qu'expérience ${ }^{1}$ (Venturini, 2012) et de l'utilisation des ressources en situation ${ }^{2}$ (Morin, 2006).

La figure 1 présente ce triple postulat de départ ${ }^{3}$.

- Figure 1. L'agir-adéquatement-en-santé

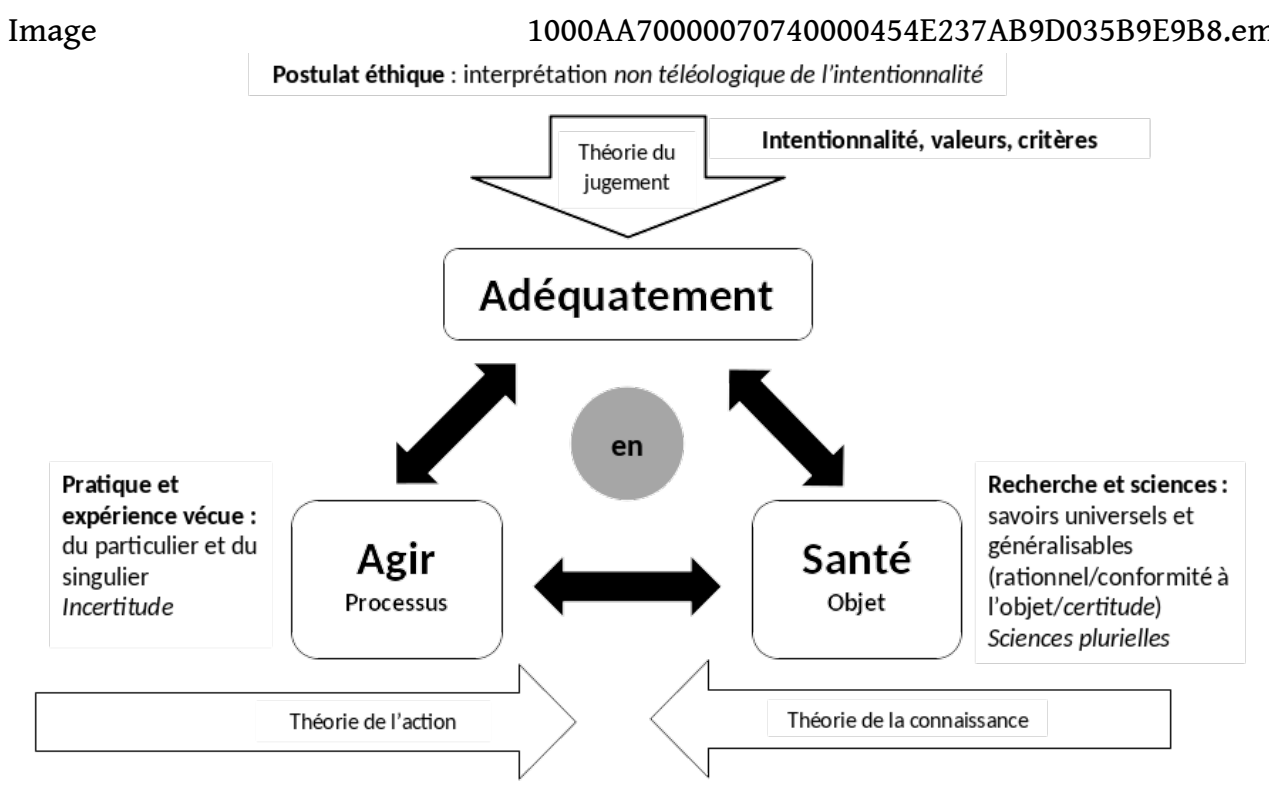

Postulat épistémologique : le savoir comme pratique Postulat anthropologique : ouverture à la pluralité des savoirs

Plus précisément sur cette figure nous observons :

- un pôle en bas à droite centré sur les savoirs stabilisés, universels ou généralisables faisant référence aux résultats de toute forme de recherche en tant que domaine de production (savoir, concept, culture, œuvres d'art, essences, théories...) et ceci relativement à la définition de la santé mobilisée. Ce pôle fait explicitement référence aux théories de la connaissance.

- un pôle en bas à gauche centré sur l'expérience vécue ou la pratique faisant référence au domaine des métiers en tant que domaines d'activités et d'actions (enseignant, étudiant, chercheur, patient, technicien, infirmier, médecin, psychologue, ostéopathe et autres soignants, ...). Ce pôle fait explicitement référence aux théories de l'action en éducation.

- un pôle en haut centré sur l'intentionnalité de l'agir humain permettant de questionner le sens ou la valeur de l'agir-en-santé au regard des deux autres pôles. Ce pôle fait explicitement référence à la dimension axiologique de l'agir et aux théories du jugement.

11 Selon l'approche pragmatiste les trois théories interagissent. Ainsi, la théorie de la connaissance indique que connaître est une action et que l'action précède la théorie (Eoyang \& Menning, 2019). La théorie de l'action réfère à la connaissance ne fût-ce qu'en mobilisant des connaissances validées par la science. Enfin, la théorie du jugement, toujours mise en contexte, est liée aux deux autres, notamment en référence 
à l'approche pragmatiste, où la "valuation" - cf. "La formation des valeurs" (Dewey, 2008 ; Foucart, 2013) est toujours située.

\section{Objectif et méthode}

12 Au regard de la figure 1 qui nous sert de fil conducteur, il nous semble pertinent, d'une part, d'expliciter le rôle d'une taxonomie dans les processus de reliance entre les différents pôles de la figure. De l'autre, d'analyser dans quelle mesure une taxonomie peut aider à élargir le spectre des activités et des savoirs pertinents pour un «agiradéquatement-en-santé ».

13 À cette fin, nous présentons une taxonomie élaborée dans le cadre de la démarche de l'approche par compétences intégrée (APCi) (Parent \& Jouquan, 2015) en la confrontant à une grille d'analyse systémique permettant d'aborder ces différents niveaux de questionnements et leurs implications en termes de possibilités d'ouvertures épistémologiques.

14 Sur ce point, la logique des compétences, caractéristique d'un «tournant pragmatique » opéré par les sciences sociales et cognitives notamment en ce qu'elle insiste sur la nécessaire prise en compte du contexte et des acteurs dans la démarche d'apprentissage, permet de formaliser une "pragmatisation» des études en santé résolument centrée sur la pratique professionnelle (en passant de l'objet "savoir » à l'objet «compétence»). Il nous semble cependant nécessaire d'en renforcer les méthodes tout en assurant un approfondissement de ses perspectives épistémologiques.

15 C'est l'intérêt de l'APCi de dépasser en partie le formalisme voire le mentalisme des approches par compétences (APC) inscrites dans les courants constructivistes et socioconstructivistes au profit d'une approche plus résolument contextuelle (au sens fort du terme, c'est-à-dire se centrant sur l'expérience, le vécu, l'agentivité et le contexte). Mais également de dépasser un certain réductionnisme lié à l'emprise d'une logique d'efficacité et de transparence qui retire toute légitimité à ce qui ne relève pas de l'apprentissage de compétences objectivement évaluables

\section{La notion de taxonomie en éducation}

La notion de taxonomie en éducation est, comme celle de compétence, sujette à de nombreuses controverses autant dans ses définitions que dans ses usages.

Pour certains, l'usage d'une taxonomie pour structurer un programme de formation par compétence représente un retour en arrière vers une pédagogie par objectif (PPO) de nature behaviouriste ou comportementale (Poumay \& Tardif, 2017).

Pour notre part et de manière plus générale, la perspective de l'APC s'inscrit bien dans la progression offerte par la PPO (De Ketele \& Delory, 2000), à savoir cet acquis considérable que l'objectif pédagogique devrait être d'abord centré sur l'activité (et non pas le savoir) permettant une valorisation de l'activité (du Sujet) relativement au savoir (défini sur une base réaliste et positive et extérieur au Sujet). Il ne s'agit cependant pas de réduire cette activité à la seule dimension de comportement et à un morcellement de tâches observables. 
19 C'est bien ce à quoi fait référence la notion de compétence. Celle-ci, dans notre conception, se veut contributive d'une perspective de capabilité du Sujet, autrement dit du développement par ce dernier d'un certain pouvoir d'agir (Monnet, 2008).

Le défi est ainsi de développer des taxonomies qui ont l'ambition d'aider à structurer et évaluer les programmes d'études ou les apprentissages dans une perspective de reliance, telle que ce concept est développé dans la pensée de Morin ${ }^{4}$ (Morin, 2004), et pas uniquement de séparation ou de fragmentation du Sujet (agent) ou du Monde (savoirs). C'est ce que nous voulons signifier en passant de l'APC à l'APCi .

\section{Présentation de la taxonomie de l'Approche par compétences intégrée (APCi) au regard des quatre critères de la systémique de Durand}

21 Nous utiliserons tout au long de la présentation de cette taxonomie et, à titre d'analyse et de discussion de celle-ci, les quatre critères qui définissent la notion de systémique selon Durand (2010): la globalité, l'organisation, l'interaction et la complexité. Cette démarche permet d'appréhender méthodologiquement, et sans trop la réduire, la complexité du réel auquel nous devons faire face, dans une visée pédagogique intégratrice et que l'on souhaite émancipatrice.

22 Avant d'entamer cette présentation, clarifions le concept de système en reprenant sa définition donnée par de Saussure cité dans Durand (2010) : le système est « une totalité organisée, faite d'éléments solidaires ne pouvant être définis que les uns par rapport aux autres en fonction de leur place dans cette totalité ». D'autres auteurs (cités dans ce même ouvrage de référence) adjoignent une dimension de complexité et d'interaction dynamique à cette définition, tandis qu'Edgar Morin la définit ainsi : "Unité globale organisée d'interrelations entre éléments, actions ou individus ».

En référence à cette dernière définition, il nous faut montrer que la taxonomie de l'APCi représente un objet-système qui rend compte de l'unité globale de l'agir-en-santé, et dont il est possible de concevoir l'organisation interrelationnelle de ses éléments dans une visée systémique.

\section{Critère de globalité}

Dans sa définition de la globalité, Durand (2010) cite Von Bertalanffy le premier à montrer qu'un système est un tout non réductible à ses parties. Ainsi il définit la globalité par la notion reprise du grec holos, tout, en explicitant que le tout est davantage qu'une forme globale, il implique l'apparition de qualités émergentes que ne possèdent pas les parties.

Dans l'analyse proposée, le critère de globalité appliqué à la taxonomie de l'APCi et à notre objet-système qu'est l'agir-adéquatement-en-santé, est argumenté par rapport au sujet et par rapport au contexte. 


\section{Par rapport au Sujet}

26 À l'origine l'APC se base sur l'analyse de l'activité réellement prestée à partir d'une démarche de didactique professionnelle souhaitant reconnaître et valoriser les connaissances liées à l'expérience professionnelle.

27 Fondée sur une base similaire, l'APCi veut rendre compte de la façon la plus juste possible de l'ensemble de l'activité déployée au sein de ces différentes expériences professionnelles vécues selon les contextes d'actions.

28 Ainsi en fonction de l'ampleur de l'activité considérée ou perçue, certaines parts de l'activité du professionnel de la santé, seront reconnues comme activités didactiquement intéressantes à développer dans l'institution éducative.

29 Étant, comme dans le cas de la PPO, centrée sur l'activité, celle-ci (l'activité) est traduite (et hypothétiquement limitée) dans le langage par le verbe d'action.

30 L'ampleur de l'activité considérée dans le cadre de l'APCi est la plus vaste possible afin d'essayer de ne rien nier du réel manifesté par le professionnel dans son agir et, par le langage (qui traduit et pourrait ainsi réduire cet agir), dans le verbe d'action.

31 En réintégrant et en réamorçant notre compréhension des savoirs et connaissances depuis et en l'agir, cette réduction devient, paradoxalement, sur le plan de la méthode, une radicalisation de l'ouverture intégratrice recherchée si on s'en réfère à l'approche du philosophe Ernst Cassirer ${ }^{5}$ (1957). Il s'agit donc, sur un plan méthodologique, d'identifier ces parties, que l'on nommera catégories de l'agir, traductibles en verbes d'action.

32 C'est ce que propose la taxonomie de l'APCi qui répertorie, en prenant en considération le visible et l'invisible traduisibles dans les verbes d'action du langage véhiculé dans un contexte culturellement situé, huit catégories que nous nommons également domaines d'activités aptes à rendre compte de la richesse de l'agir-en-santé, illustrées dans la figure suivante (Figure 2) ${ }^{6}$. 
Figure 2 : Les huit domaines de l'activité qui rendent compte de la globalité de l'agir-en-santé

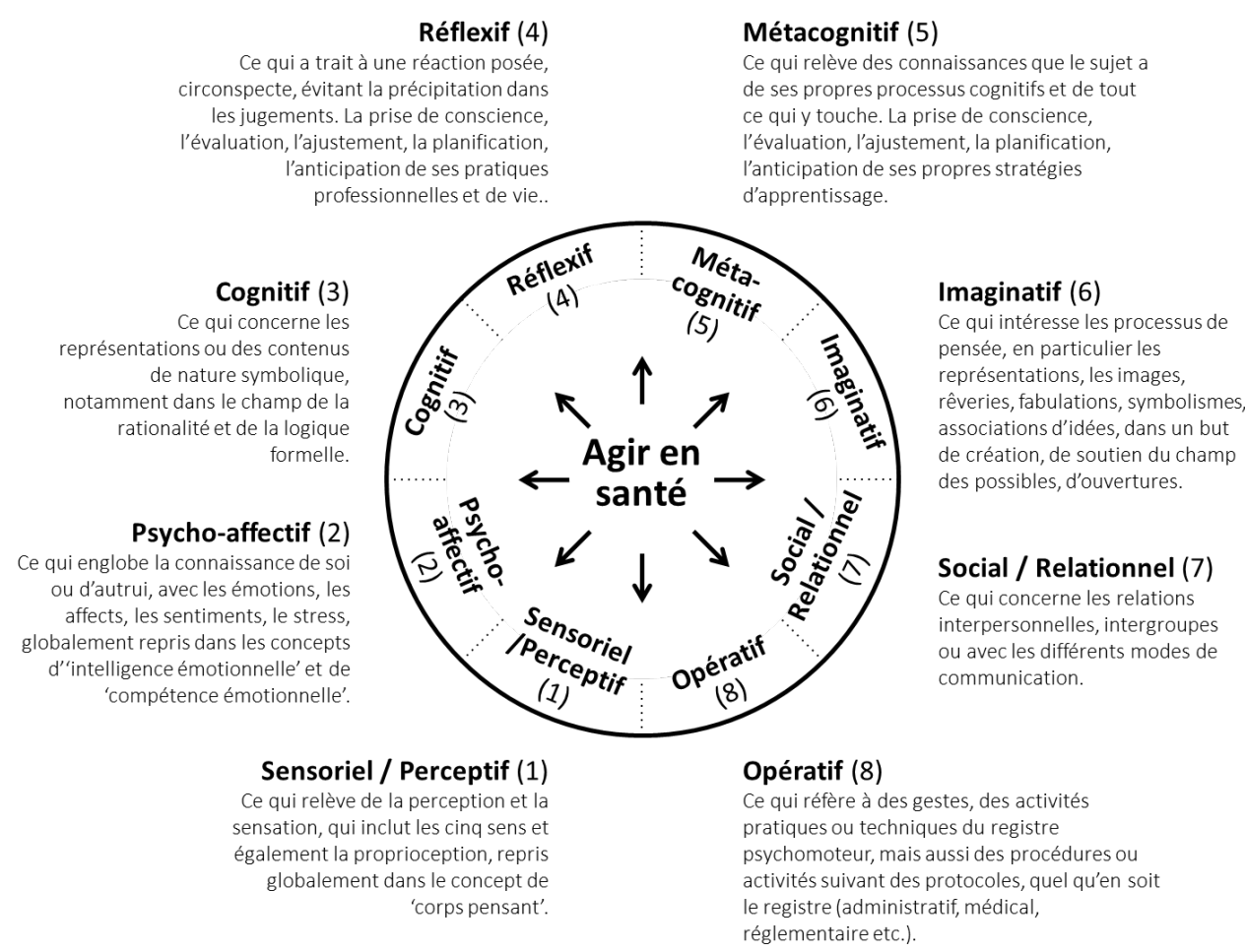

L'ordre des catégories, en l'absence de hiérarchie, est explicité avec le critère organisation.

S'intéresser à l'ensemble de l'activité humaine en essayant de ne pas en nier une part, participe d'un processus de reconnaissance du potentiel de globalité de l'agir en (situations professionnelles de) santé.

La phrase de Pascal selon laquelle il est «impossible de connaître les parties sans connaître le tout, non plus que de connaître le tout sans connaître particulièrement les parties " souligne ce processus d'interaction entre chacune de ces catégories et leur mobilisation nécessairement plurielle en situations.

N'est-il alors pas réducteur par rapport à ce critère de globalité, d'observer que dans de très nombreux contextes de formation en médecine, on développe de façon formelle et sur de longues années, principalement un "agir-en-santé » centré sur une ou deux catégories, cognitive ou réflexive, faisant appel à des activités mentales limitées à des verbes d'action tels que "mémoriser ", "se souvenir ", " connaître ", " raisonner ", "répéter ", par exemple? Si le point d'entrée du curriculum est le savoir, avec une table des matières ou un contenu explicite de savoirs identifiés en rapport avec des champs de recherche en bioclinique et en sciences fondamentales notamment, l'activité principale que l'étudiant sera amené à développer sera un "agir-connaître », et l'institution facultaire aura l'illusion d'une maîtrise des savoirs par le biais d'une assurance quantitative de savoirs déclaratifs.

Dans ce cas et sur une base systémique, inévitablement la capacité humaine favorisée par l'institution éducative se limitera à "ce qui a été mis en activité ", à ce qui aura "été agi », tout en se satisfaisant d'une définition de l'intelligence faisant référence étymologiquement à l' "âme intelligible » à laquelle Aristote nous convie en tant qu'être humain. 


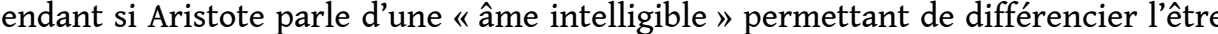
humain de l'être de nature (plante) ou de l'être vivant (animal), il le fait sans discontinuité, en totale intégration, avec l' "âme sensible " et l' "âme végétative ", l'une n'allant pas sans l'autre au risque d'une rupture du tout, de la globalité, de la perte radicale d'une vision holistique.

C'est ce que reproche Damasio (2006 p. 338-339) à Descartes :

L'idée d'un esprit séparé du corps a, semble-t-il, également orienté la façon dont la médecine occidentale s'est attaquée à l'étude et au traitement des maladies. La coupure cartésienne imprègne aussi bien la recherche que la pratique médicale. Par suite, l'impact psychologique des maladies affectant le corps proprement dit (ce que l'on appelle les maladies réelles) n'est généralement pas pris en compte, ou seulement envisagé dans un second temps. Le processus inverse, la façon dont les problèmes psychologiques retentissent sur le corps, est encore plus négligé. N'est-il pas curieux de penser que Descartes, qui a véritablement contribué à modifier le cours de la médecine, lui a fait abandonner l'approche holiste, dans laquelle l'esprit et le corps ne faisaient qu'un, approche qui avait prévalu d'Hippocrate à la Renaissance? Aristote aurait été bien mécontent de Descartes, s'il l'avait su.

Ainsi, dans une perspective systémique, c'est l'ensemble de notre agir, également l'agir sensible, émotionnel, corporel, végétatif qui concourt à résoudre une situation ou un problème, celui-ci pouvant aussi bien être la santé de l'étudiant lui-même. Penser que seule une partie de notre agir, agit, -est-agie-, est un leurre de l'esprit. Mais penser que l'ensemble agit, -est-agi-, et parfois de façon dysfonctionnelle nécessite un travail analytique de séparation des éléments afin de mieux travailler à l'harmonie de leur reliance.

C'est ce que les neurosciences cognitives et affectives nous apprennent. Les approches neuroplastiques, par exemple, exigent du patient une participation active à tous les niveaux : mental, neurologique et corporel, corps et esprit s'associent pour guérir le cerveau. Comme le souligne Norman Doidge (Doidge, 2015, p. 21), « Hippocrate, le père de la médecine scientifique, voyait déjà dans le corps le guérisseur suprême, et considérait médecin et patient comme alliés travaillant avec la nature afin d'aider l'organisme à activer ses propres capacités ».

41 Ainsi, " ressentir », " méditer ", " voir ", « imaginer ", " goûter ", " percevoir », « jouer », « souffrir », « toucher », « digérer ", « mastiquer », « marcher ", « aimer ", etc. apparaissent de mieux en mieux comme des verbes d'action qui représentent une activité humaine fondamentale pour résoudre les problèmes que l'être humain, patient ou professionnel, est amené à rencontrer quotidiennement dans son vécu ou sa pratique professionnelle. Il ne s'agit plus de les séparer des activités cognitives plus classiques, mais au contraire de les considérer comme des ressources à développer (van Grunsven, 2015). Nos activités cognitives relatives au développement personnel ou au développement professionnel ne sont pas séparées d'une ligne de démarcation nette, forme de frontières qui existeraient entre des parties de notre « corps pensant » (van der Kolk, 2018).

De dissidentes, ces activités deviennent, notamment par les recherches en anthropologie et en neurosciences, pleinement existantes sur un plan scientifique, et progressivement mieux reconnues dans divers champs d'application tels que celui de l'éducation médicale.

43 La reconnaissance de la reliance émotion-cognition nécessaire dans de nombreuses situations professionnelles (décision ; accompagnement ; leadership ; etc.) est à l'œuvre 
à travers l'émergence d'une intelligence émotionnelle corroborée par le concept de compétence émotionnelle en éducation médicale (Parent, Jouquan, Kerkhove, Jaffrelot \& De Ketele, 2012 ; Parent \& Jouquan, 2019 ; Arora, Ashrafian, Davis, Athanasiou, Darzi, \& Sevdalis, 2010). Dans un curriculum par compétences, celle-ci se traduit nécessairement en activité, -ou agir-, pour l'être humain sous forme de verbes d'action centrés sur la dimension émotionnelle de l'agir ("identifier ses émotions" « reconnaitre ses facteurs de stress »; "s'ouvrir à ses affects »; «favoriser le lâcherprise » etc.) telle que nous la développons, de façon critique au regard de certaines logiques simplificatrices et managériales actuelles (De Funès, 2019), dans diverses contributions (Jouquan, Parent, Mathé \& Burucoa, 2019).

Il nous aura fallu l'argument scientifique corroboré par l'imagerie en provenance des neurosciences pour venir paradoxalement confirmer les limites d'un raisonnement - et d'une intelligence - purement cognitifs et rationnels qui prévalent depuis notre modernité cartésienne (elle-même bien installée sur un platonisme ancien) et, sur laquelle, par ailleurs, s'est élaborée notre épistémologie scientifique classique, positiviste.

L'APC apparaît non seulement comme une opportunité permettant de se centrer sur la pratique par le biais de l'activité, mais encore davantage, comme un potentiel levier de reliance de nos "âmes multiples ", végétative, sensorielle et intelligible, pour autant que l'on fasse le choix d'une vision holistique de l'agir humain. Sur le plan éducationnel, l'enjeu devient alors d'opérationnaliser une telle perspective ce qui induit notamment le besoin d'élaborer des taxonomies intégratrices favorables à cette perspective de reliance.

En résumé, il s'agit de procéder à une démarche de didactique professionnelle qui part de l'analyse de l'activité du professionnel de la santé, activité traduisible en verbes d'action et qui inclut tous les phénomènes expérimentés par le sujet ou, préférentiellement, « agis » par le Sujet. Ces phénomènes proviennent de l'expérience vécue du «mouvement exprimable», pas seulement du seul "qui se voit» (prescrire, toucher, accompagner etc.) mais aussi de celui «qui ne se voit pas» (raisonner, apprécier, sentir, aimer etc.). C'est-à-dire un mouvement du comportement visible ou mesurable mais aussi de la pensée, mouvement qu'on dira à la fois visible et invisible, incluant autant le champ procédural que celui de la cognition, des affects ou de l'imaginaire. La taxonomie offre une grille d'analyse ouverte à cette fin.

\section{Par rapport au contexte}

47 Par ailleurs, l'activité du professionnel de la santé est liée ou déterminée par un ensemble de facteurs contextuels et culturels en interactions (contraintes, normes, opportunités et influences multiples). La notion de pratique professionnelle émerge, en tant que devenir, d'un "agir-en-santé », c'est-à-dire un "agir-en-situationsprofessionnelles-de-santé » faisant nécessairement référence aux contextes spécifiques de l'action.

Le critère de globalité devrait ainsi conséquemment se compléter par une délimitation de l'objet-Monde dans lequel agit le Sujet, tout comme nous avons délimité l'agir de l'objet-Sujet dans la figure 2 .

En effet, la notion même de pratique professionnelle ne peut s'expérimenter en dehors d'une temporalité suffisamment longue liée à des contextes concrets et particuliers qui 
sont autant de situations professionnelles qu'il est nécessaire de recenser pour identifier le profil professionnel en développement. Ainsi les activités que le professionnel sera amené à développer seront directement "inter-agies", selon le principe du ruban de Möbius, par ces situations et contextes, tandis que ceux-ci (contextes) ne restent pas immuables, comme tout paysage, ils se modifient avec la présence en mouvement de l'humain (Roger, 1997).

Une "situation professionnelle ", conjonction d'un rôle professionnel et d'un contexte d'exercice professionnel, se définit en rapport avec un système de santé donné. Ainsi l'objet-système représenté par la taxonomie se complète d'une interaction contextuelle assurant une ouverture environnementale à celui-ci qui devient ainsi un système ouvert.

Ce niveau contextuel participe d'une catégorie supplémentaire, celle de la synthèse, par contraste avec celle des activités dont la logique est analytique. Cette catégorie (synthèse) est développée dans la figure 3 et est à mettre en relation notamment avec le critère d'organisation.

52 Ainsi, à l'étape de planification, la globalité tient compte, à la fois, de l'ensemble de l'agir-en-santé du Sujet et de l'ensemble des situations et contextes auxquels le professionnel peut être, très concrètement, confronté.

53 Ce sont ces mêmes situations qui vont déterminer, en grande partie, les savoirs spécifiques à mobiliser en fonction de chaque profession. L'ouverture-Sujet, c'est-à-dire sur les dimensions de l'individualité représentées par les domaines d'activités et, de l'autre, l'ouverture-Monde représentée par des contextes d'exercices professionnels diversifiés, permettent de 'faire entrer' dans le curriculum des champs de savoirs pluriels ouvrant à la perspective d'une science plurielle.

C'est à ce niveau, en partie, que se joue la réponse à la problématique méthodologique de reliance théorie-pratique entre savoirs et activités professionnelles, ce qu'on nommera en lien avec les deux pôles inférieurs de la figure 1 «la reliance savoirsprofession ".

De cette logique de catégorisation qui se fonde sur une perspective anthropologique de reliance de l'être humain, émerge une « structure taxonomique » qui peut s'apparenter, - forme d'isomorphisme-, avec celle d'un "Sujet transcendantal». Le Sujet transcendantal étant défini comme a priori, universel et conditions de possibilité du Sujet empirique individuel se singularisant dans l'expérience vécue, tel que mobilisé en philosophie chez Kant et repris en phénoménologie par Husserl.

Nous faisons l'hypothèse ambitieuse, certes, de ce parallélisme en référence au fait que ces deux philosophes étaient avant tout des épistémologues visant à des formes universelles de la connaissance, Kant limitant son Sujet transcendantal (catégories a priori) à la physique newtonienne, tandis que Husserl visant à définir les structures universelles de l'agir, englobant de façon beaucoup plus large (que ne le fait Kant) et le Sujet (psychologique) et le Monde. Nous retrouvons ainsi par cette forme de corrélation (phénoménologique et taxonomique) les fondements structurants d'une perspective épistémologique qui vise à une reliance "corps-esprit-monde » en référence à Paul Valéry cité par Jean-Louis Le Moigne (Le Moigne, 2012, p. 54) ouvrant à une "reliance théorie-pratique » telle que nous cherchons à la mettre en œuvre (fig. 1).

57 En faisant référence au Sujet transcendantal et surtout dans sa relecture husserlienne, en le caractérisant par son rapport dynamique avec le Monde, son environnement etc. nous renvoyons notamment aux travaux de Berthoz et Petit (Berthoz \& Petit, 2006). En 
effet, en mentionnant explicitement des kinesthèses dans la phénoménologie de Husserl, ceux-ci définissent le corps propre comme un "échangeur du subjectif en objectif et réciproquement ", rejoignant par là notre préoccupation systémique.

Il découle de cela que la compétence n'existe jamais à priori et de façon universelle, elle ne peut être que située et singulière car propre au "Sujet empirique individuel». Il serait donc impossible de nommer des compétences dans un référentiel de compétences contrairement aux ressources (dimensions de l'individualité) et aux situations (contextes).

En effet, c'est par le biais d'une articulation entre ces deux types de catégorisation, analytique et synthétique, qu'a lieu le processus complexe de transformation et du Sujet et $\mathrm{du}$ Monde, nous permettant de rapprocher l'agir-en-santé en tant qu'expérience et pratique de celle de l'Art. Car

L'art est non pas l'émanation spirituelle éthérée d'une muse céleste lointaine, mais une excrétion incarnée, expressivement épurée, des énergies naturelles présentes dans nos transactions vivantes avec notre environnement naturel et culturel, orientée vers un accomplissement supérieur de la vie. (Dewey, 2015. p.13-14).

C'est ce que les critères suivants, l'organisation, l'interaction et la complexité, vont nous permettre d'approfondir, le critère de globalité ayant par ailleurs été proportionnellement nettement plus développé car il définit en quelque sorte le terrain de jeux des suivants.

\section{Critère d'organisation}

61 Selon Durand (2010, p.10),

L'organisation peut être considérée comme le concept central de la systémique. Cette organisation est d'abord un agencement de relations entre composants ou individus qui produit une nouvelle unité possédant des qualités que n'ont pas ses composants. Pour mieux comprendre, on peut donner l'exemple des isomères qui sont des composés chimiques de même formule et de même masse, mais ayant des agencements structurels différents et, par voie de conséquence, des comportements différents.

62 Le critère organisation est un critère qui définit toutes taxonomies puisque celles-ci sont élaborées à partir de catégories explicites et mutuellement exclusives (éléments ou composants) qui se structurent selon un certain agencement. Les taxonomies en éducation participent en général à la fois à structurer des éléments (ou composants) définis en termes d'objectifs d'apprentissages permettant de les décrire, mais également d'en analyser la structure et/ou de la réguler.

63 L'agencement et le choix des composants sont tous les deux des éléments critiques dans la détermination de notre « objet-système ». En effet chacun des éléments est contributif des autres en fonction de l'agencement de l'ensemble.

Si les huit catégories de l'« agir-en-santé » dans la figure précédente (Fig. 2) décrivent les composants de notre « objet-système » partant des postulats de la centralité de l'action et d'un agir global (Fig. 1), un agencement est déjà bien présent permettant de considérer que nous avons, d'ores et déjà, abordé le critère d'organisation.

Pour ce qui est de l'ordre préétabli des catégories (logique analytique), cet agencement n'a pas de conséquence fonctionnelle étant donné son absence de hiérarchie. Celle-ci est en effet conceptuelle, manifestant le sensoriel et l'émotionnel comme premier et 
cause des domaines suivants, notamment celui de l'imaginaire, tout en reconnaissant des boucles de rétroactions perpétuelles entre les diverses dimensions de l'individualité.

En ce qui concerne notre taxonomie APCi nous avons, à ce stade-ci, argumenté deux plans logiques différents dont les composants de chacun des plans devraient être de nature identique :

- Celui des activités traduisibles en verbe d'actions, qui se structure en domaines d'activités, nommé l'approche analytique de la compétence.

- Celui des contextes traduisibles en situations professionnelles nommé l'approche située de la compétence.

67 Cette éventuelle contradiction logique dans une perspective rationnelle (taxonomique, catégorielle) qui permet de reconnaître la dimension chaotique du «toujours particulier » est un argument en faveur d'une réelle prise en compte de la perspective systémique qu'on souhaite favoriser.

Une autre catégorisation (plan logique) va venir complexifier cette première structuration, il s'agit des niveaux de maîtrise pour chacune de ces catégories (Figure 3).

Cette figure peut être regardée en trois dimensions : le centre de la figure exprime la profondeur (les huit domaines qui sont toujours présents dans toute pratique professionnelle (expérience)); en allant vers la périphérie, on développe des compétences de plus en plus complexes (où l'agir-adéquatement-en-santé s'inscrit dans des situations de plus en plus inédites et tend à produire de nouvelles connaissances et de nouveaux schèmes d'action). De plus, cette même figure tente de rendre compte des potentialités d'ouverture et de développement de l'activité. L'objectif est l'intégration progressive d'un agir-en-santé quelle que soit la part (parcellaire ou plus globale) de l'activité développée. 
Figure 3 : L'organisation des niveaux de maîtrise et l'intégration des huit domaines de l'activité dans l'apprentissage de l'agir-en-santé.

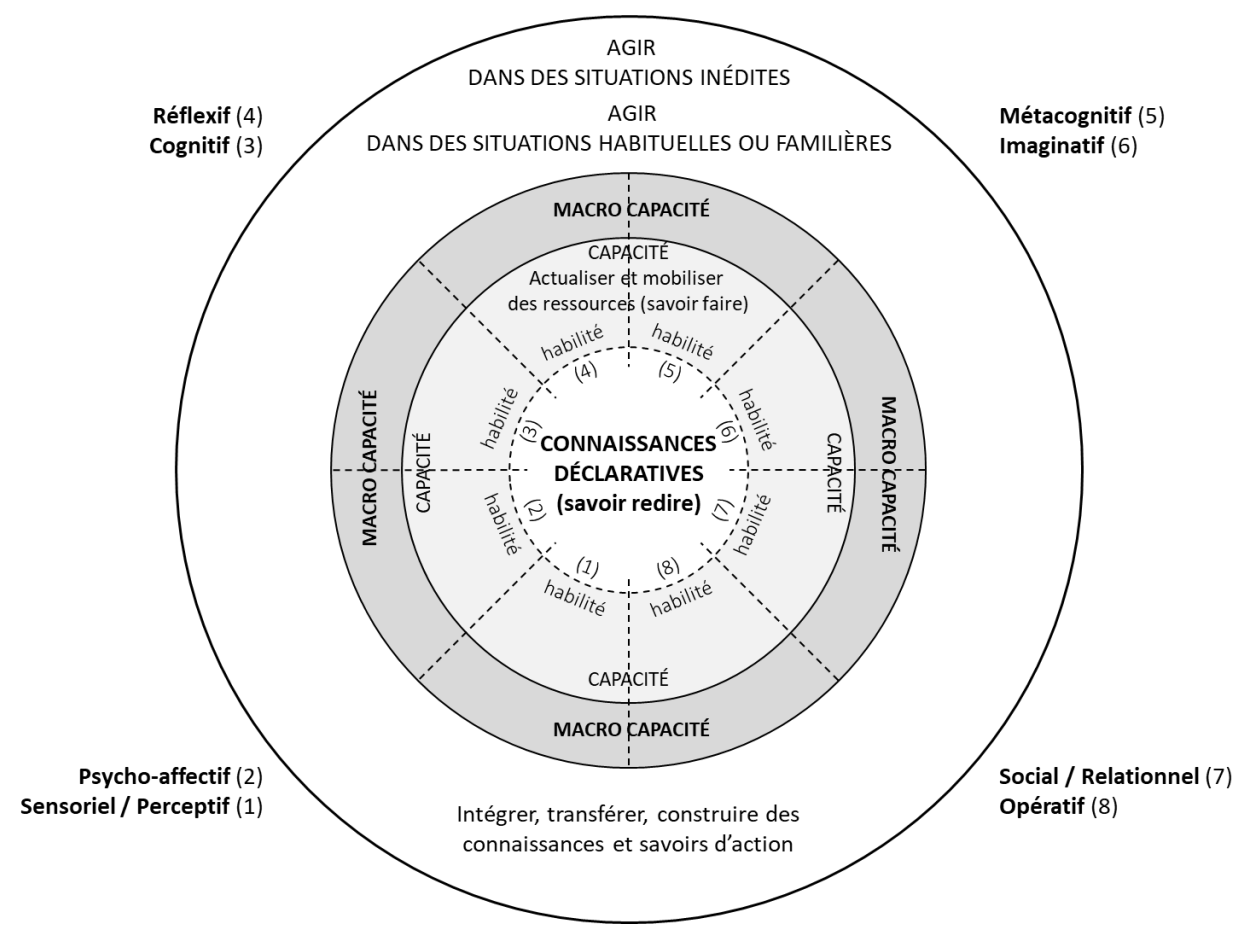

En partant de cette figure, nous pouvons mieux comprendre l'agencement global de la taxonomie et son lien avec ce plan logique supplémentaire si l'on considère divers points.

Ainsi, la séparation entre domaines devrait dessiner des lignes de plus en plus pointillées au point de disparaître complètement dans le niveau de maîtrise «agir-ensituation(s)».

Gardons à l'esprit qu'au niveau de la séparation entre savoir-redire et savoir-faire, le savoir-faire fait toujours référence à l'une ou l'autre connaissance déclarative. Il arrive souvent (notamment avec l'habitude) que l'on maîtrise un savoir-faire sans plus savoirredire la ou les connaissance(s) déclarative(s) sous-jacente(s).

Dans le savoir-faire, il y a des niveaux de complexités très différents, depuis des habiletés simples (refaire une démarche apprise) à des capacités, relayées elles-mêmes par des macro-capacités. Ceci est fonction de la variété plus ou moins expérimentée des paramètres des situations rencontrées.

Pointons aussi que dans l'agir en situation, les paramètres à envisager sont nombreux. Il faut sans doute distinguer des situations habituelles ou familières (les paramètres à prendre en considération sont multiples, mais le soignant est habitué à en tenir compte) des situations qui ne sont plus habituelles (soit un changement de contexte professionnel extérieur ou intérieur, soit des éléments non habituels entrent dans la situation, celle-ci inclut le Sujet et le Monde). Dans le premier cas, il y a mobilisation/ intégration des acquis en situation (connaissances et schèmes d'action connus) de pratique professionnelle familière ; dans le second cas, il y a mobilisation/intégration/ transfert d'acquis et construction de nouveaux schémas d'action et connaissances sous l'effet de la confrontation entre éléments nouveaux et anciens. 
75 La construction de connaissances nouvelles (intégration et articulation de nouveaux éléments dans une connaissance nouvelle plus complexe) ou de schèmes d'action nouveaux (également par intégration et articulation de schèmes d'action inédits avec les schèmes d'action antérieurs) nécessite d'être confrontée à de nouvelles situations. Ceci est à la base du développement professionnel, conçu comme "professionnalité émergente ", jamais achevée (Jorro \& De Ketele, 2011).

Enfin, retenons qu'il s'agit d'une approche située de la compétence, où les niveaux de maîtrise et de développement dépendent beaucoup des paramètres des situations rencontrées (ou à faire rencontrer si l'on se place du point de vue de l'apprentissage).

Une comparaison avec la taxonomie «savoir ; savoir-faire ; savoir-être » fréquemment mobilisée dans des contextes d'APC, permet également de signaler des confusions qui peuvent exister entre des composants (de plans logiques différents) dont l'organisation des liens n'a pas été rendue suffisamment explicite ne favorisant pas une clarification quant au concept de compétence mobilisé (nous en avons rendu compte dans un précédent essai) (Parent \& Jouquan, 2015, p. 104).

78 C'est seulement à partir de telles clarifications qu'un débat critique quant à l'usage du concept de compétence peut être engagé. Nous le jugeons nécessaire car le concept de compétence dans sa mouvance actuelle permet(trait) selon certaines conditions une bascule vers la centralité de l'action et vers une épistémologie de la pratique, seule à même de réhabiliter radicalement le particulier et le singulier. Une telle possibilité donnerait la voie d'une articulation entre les deux pôles inférieurs de notre figure 1 , réhabilitant l'universel (Savoir) et le singulier (Action) dans une perspective collective commune.

\section{Critère d'interaction}

79 L'interaction est fortement déterminée par les possibilités d'organisation des composants entre eux, c'est-à-dire leur agencement. Ce que nous développons ici est partiellement redondant par rapport aux développements précédents, mais permet d'argumenter autrement la perspective systémique et surtout son potentiel de complexité. Ainsi, l'interaction peut prendre des formes plus ou moins complexes, depuis le simple lien de causalité directe, jusqu'aux relations d'une grande variété et subtilité entre éléments du système (Durand, 2010).

En effet, une particularité complémentaire déjà énoncée avec le critère de globalité permet de faire émerger la fonction combinatoire de notre "objet-système ». Il s'agit de la possibilité de reconnaître la "fusion» entre les catégories relatives aux huit domaines d'activités pour le niveau de maitrise en contextes authentiques qui correspond au niveau de l'« agir en situation(s) ». Comme la notion de situation fait référence en ce qui nous concerne aux situations de santé nous parlons d'«agir-ensanté».

81 Cet «agir-en-santé » reflète la compétence comme nous l'avons argumenté sur un plan épistémologique. En effet, la compétence devient la manifestation singulière d'une mobilisation de ressources à dimensions multiples, éclectiques, par une personne, un Sujet-Individu-Agent, dans un contexte toujours particulier faisant référence sur un plan didactique à la notion de situations professionnelles. 

pluriels et particuliers. Cependant cette reliance pour ne pas être réductrice quant aux savoirs à mobiliser devrait être pensée en lien très direct avec le critère de globalité. C'est ce dernier qui va donner corps (dans un sens concret et matériel) à la pertinence et l'efficience d'un curriculum. Seule la considération, d'une part, de l'ensemble, -de la plus vaste ampleur-, de l'activité dans le sens de l'agir du professionnel («agir-en-santé ») et, d'autre part, de l'ensemble des situations professionnelles («agir-en-santé ») rencontrées (parfois de façon prospective) dans un système de santé et de société particulier, permet le développement d'un réel corps en devenir professionnel («agir-ensanté »), qui s'incarne temporellement dans ce qui a été défini par le biais de la taxonomie comme l'" agir-en-santé ». 
88 Dans le cadre de l'APCi, l'intégration est notamment assurée par l'usage de la taxonomie pour, d'un côté, planifier les objectifs pédagogiques à partir de l'activité professionnelle (traduction didactique) et, de l'autre, catégoriser les phénomènes d'apprentissage en prévision de la transposition pédagogique. La correspondance entre les deux catégorisations est immédiate et favorable par ailleurs à la problématique du transfert des apprentissages.

Ainsi ce sont autant les deux dimensions, analytique et située, qui vont permettre de travailler la «reliance théorie-pratique ». En faisant référence à une taxonomie centrée sur l' «agir» (plutôt que le savoir que sous-tend le concept), c'est-à-dire centrée sur le verbe d'action («soigner » plutôt que «le soin »; «penser » plutôt que «la pensée »; «vivre» plutôt que «la vie» etc.) nous intégrons directement la question de l'autonomie du Sujet au regard de la notion de capacitation que nous définissons en lien avec sa pratique professionnelle et la mise en acte de son « agir-en-santé ».

C'est le cas pour toutes les taxonomies centrées sur l'activité, celle-ci étant déclinée sous forme de faculté, d'aptitude, de capacité ou d'habileté. Toutefois ces capacités ne sont pas en suspension, elles sont ancrées dans des situations réelles qui délimitent et impliquent un champ de savoirs. La "reliance théorie-pratique » intervient en grande partie à cet endroit, dans une circularité, entre l'approche analytique et l'approche située de la compétence.

91 Ainsi, la structure même du curriculum (critère d'organisation) devrait favoriser ce critère d'interaction. C'est une des conditions nécessaires à l'intégration «théoriepratique » qui se joue au moment de l'élaboration des programmes.

\section{Critère de complexité}

Toujours en référence au cadre d'analyse systémique mobilisé, la complexité d'un système tient au moins à trois séries de causes (Durand, 2010, p. 11) :

- «Celles inhérentes à la composition même du système, au nombre et à la caractéristique de ses éléments et surtout de ses liaisons ;

- Celles provenant de l'incertitude et des aléas propres à son environnement ;

- Celles enfin qui tiennent aux rapports ambigus entre déterminisme et hasard apparent, entre ordre et désordre »

Le critère complexité rend compte de la nécessaire collaboration entre tous les critères de la systémique mobilisés.

La taxonomie de l'APCi, comme n'importe quelle taxonomie n'est pas neutre au regard des objectifs pédagogiques qu'elle détermine. Chaque taxonomie a un mouvement (dans le sens d'un drapé, d'une couverture sur le Sujet-Monde) d'une ampleur variable, plus ou moins vaste (globalité), essentiellement déterminée par le nombre de ses éléments, leur caractéristique (organisation) et, par voie de conséquence, les combinaisons que ces éléments rendent possibles (interaction).

De nombreuses taxonomies en éducation réduisent le réel, même si toute démarche méthodologique impose une forme de réduction. C'est là que le critère de complexité prend toute son importance.

96 Selon notre point de vue, il s'agit de vérifier qu'un mouvement de simplification n'amène pas à compliquer le réel en construisant des antinomies, conceptuelles ou ontologiques (pratique-théorie; développement personnel-développement 
professionnel ; « soft-skills » - « hard-skills »; privé-public; émotion-cognition ; corpsesprit; individu-collectif ; nature-culture ; féminin-masculin etc.), spéculatives ou en ne comprenant pas leurs articulations (ou les raisons de leurs éventuelles antinomies). En d'autres termes sans en clarifier l'épistémologie sous-jacente et donc la théorie de la connaissance à laquelle on se réfère et qui engage, ou non, certaines dichotomies quant au rapport au réel que l'on souhaite construire.

Ces antinomies sont notamment favorisées par des taxonomies ayant une structuration (critère organisation) trop linéaire ou l'absence de possibilité de synthèse (critère globalité et interaction). Une forme d'intuition permet de transférer cette nécessaire complexité de la taxonomie en tant qu'elle structure l'«agir-en-santé » avec la "personne ", l'individu dans toute sa nécessaire reliance, "émotion-raison" et "corpsesprit-monde".

En faisant ce choix, on postule un certain rapport au réel particulièrement intégrateur et complexe. Celui-ci présuppose l'absence de dualisme ontologique (la problématique de la séparation "corps-esprit» en philosophie de l'esprit). En effet, il intègre, au domaine cognitif, un domaine sensoriel, corporel, de même que l'absence de dualisme conceptuel (séparation "émotion-raison »). De la même manière, il inclut un domaine psychoaffectif ou émotionnel, différent du domaine relationnel ou communicationnel.

Une forme d'horizontalisme de l' "agir » apparaitt («voir» est autant valorisé que "analyser", "raisonner ", " toucher ", « sentir ", " méditer ", «créer ", « diagnostiquer » « appliquer » etc.) sans hiérarchisation de valeur entre les différentes activités. Toute activité en provenance du réel défini comme monde sensible et en mouvement possède une même valeur.

Bien sûr qu'une forte réduction participe de l'élaboration d'une taxonomie, néanmoins en tant qu'outil de planification, celle-ci peut orienter le développement de l'action dans des voies très différentes, plus ou moins réductrices en fonction de leur niveau d'intégration.

Le critère de complexité permet d'énoncer de manière plus explicite l'orientation épistémologique souhaitée. La perspective pédagogique de l'Enaction (Varela, 1988), mais également celle du pragmatisme (Deledalle, 2018) ajoutent une dimension intégrative majeure, celle du contexte dans un continuum permettant de rompre avec l'antinomie "nature-culture » et d'élargir le ruban de Möbius à une complexité essentiellement fondée sur la continuité et la circularité ontologique "corps-émotioncognition-environnement ». On peut rapprocher ce point de vue des travaux de Bozalek et Zembylas insistant sur la dimension ontologique à prendre en considération dans la recherche en éducation (Bozalec \& Zembylas, 2017).

D'où l'importance accordée aux situations dans l'APCi, celles-ci ne se limitant pas aux problèmes de santé, mais considérant les environnements de santé dans leurs dimensions psycho-sociales, interculturelles et politiques. C'est essentiellement alors que la dimension combinatoire permet de respecter le critère de complexité dans son opérationnalisation (à l'étape de la transposition pédagogique).

Pour clôturer la présentation de la taxonomie à partir des quatre critères de la systémique, nous revenons à la définition de la compétence à laquelle nous nous référons dans le cadre de l'APCi : «Une compétence est un agir complexe et singulier qui mobilise, grâce à des capacités de nature diverse (sensorielle-perceptive, psycho-affective, cognitive, réflexive, 
métacognitive, imaginative, sociale, opérative, ...) un ensemble de ressources complémentaires pour traiter de façon adéquate des problèmes, à l'intérieur de familles de situations professionnelles toujours particulières et complexes, définis au regard de contextes, de rôles, d'opportunités et de contraintes spécifiques. »

Progressivement les éléments de la définition choisie dans le cadre de l'APCi ont été argumentés par le biais de ces quatre critères de la systémique nous permettant de relever le défi de la complexité, omniprésent dans tous les systèmes qui nous entourent et nous constituent.

On retrouve bien la plupart des éléments de cette définition opérationnalisés à travers la taxonomie.

Quelques clarifications épistémologiques, dans la conclusion, permettront de mieux appréhender certains éléments de la définition qui restent encore trop peu explicités à ce stade-ci.

Néanmoins nous pouvons déjà affirmer que cette taxonomie joue bien son rôle d'« opérateur de reliance » méthodologique (Morin, 1997) permettant de tendre vers une forme de complexité du réel et de la personne.

\section{Conclusion}

La discussion avec ses ouvertures réflexives a été menée au fil de la présentation de la taxonomie. Nous la prolongeons de cette conclusion qui se lit à partir de l'analyse du pôle supérieur de la figure 1. Celle-ci aborde, sans la déterminer, le postulat 3, celui de la question de l'évaluation ou celle du jugement (la dimension axiologique de l'agir), enjeu paradigmatique des orientations épistémologiques et éthiques choisies (Jouquan \& Parent, 2013, p. 221). En effet, étant entendu qu'un tel objet (taxonomie) participe directement, par ses possibilités d'orientations épistémologiques, aux modes d'apprentissage d'un métier et de pratiques professionnelles, c'est bien une réflexion sur la praxis et donc sur l'éthique de la santé qui nous guide.

110 Si les deux pôles inférieurs de cette figure 1 peuvent être, de façon singulière et limitée, rapprochés de la Critique de la raison pure (théorie) et de la Critique de la raison pratique de Kant (avec les ouvertures phénoménologiques préalablement discutées et la nécessaire continuité entre ces deux pôles), c'est avec le concept de jugement réfléchissant, développé dans son troisième ouvrage majeur, Critique de la faculté de juger ((Kant, (1790) 1993) que nous approfondissons la question de la nécessité d'une orientation épistémologique, d'une part, vers les théories de l'action, et, d'autre part, vers celles de la créativité de l'agir telle que développée par Hans Joas (Joas, 2001).

Cependant nous sommes amenés à vérifier les spéculations potentielles dans lesquelles nous pourrions nous trouver. En effet, en quoi la question du choix d'une taxonomie nous permettrait-elle d'aller aussi loin dans l'analyse (même uniquement introductive au stade de cet essai) de tels présupposés théoriques?

112 Si la visée pragmatique dans laquelle nous cheminons nécessite des "changements méthodologiques, la création de nouveaux objets centraux d'étude et des changements lexicaux, se traduisant notamment dans l'abandon de certains couples d'opposition, incapables de montrer le caractère essentiel des dimensions pragmatiques du phénomène étudié » (Steiner, 2008), peut-on, aussi, y inclure une ouverture à la dimension axiologique de l'agir-en-santé, et alors, dans l'affirmative, comment? 
113 La taxonomie avec son fondement en didactique professionnelle nous offre les conditions de possibilité d'une mise en œuvre des deux premiers postulats (figure 1) par sa centralité sur l'action ; située ; ancrée et incarnée, et l'absence de rupture avec la dimension théorique de la connaissance. Elle quitte de ce fait la logique binaire, théorie et pratique, problématique introductive à l'ensemble de notre propos. Elle rejoint en cela les perspectives critiques de la psychologie cognitive dans ses évolutions plus récentes (théories émergentes de la cognition située et de la cognition incarnée) favorables à une rupture pragmatique, voire à une rupture pragmatiste selon Peirce ou Dewey et dont rend compte Pierre Steiner quand il interroge les sciences cognitives face à de telles évolutions de pensée (Steiner, 2008).

114 Cependant, "le défi pour les sciences humaines et sociales, dès lors qu'elles se saisissent de l'action, réside dans l'analyse de sa constitution propre, où l'on reconnaît au sujet une capacité de connaître et d'interpréter les situations et les interactions, sans pour autant renoncer à une posture scientifique distanciée, procédant par confrontation et accumulation » (Baudouin, 2001, p.8). Reconnaissant à la taxonomie APCi la possibilité d'une place ouverte et interactive à l'agir et aux savoirs pluriels (figure 1), l'enjeu en suspens est de comprendre dans quelle mesure ce cadre méthodologique (taxonomique) permet, - donne une place- à cette dimension interprétative du Sujet et du Monde, et de leurs interpénétrations.

115 Pour cela, nous revenons à la définition de la compétence qui a émergé progressivement à partir de l'analyse systématique de la taxonomie. Celle-ci (compétence) n'existe pas a priori, en tant qu'universelle, généralisable ou prédéfinie. Elle appartient à chaque Sujet empirique individuel, s'élabore en contexte et devrait permettre de rendre compte du processus transactionnel à l'œuvre, explicité par l'utilisation de la métaphore du «nœud de Möbius». Ainsi, évaluer ou juger une personne ou une situation (problème), c'est comprendre, d'abord, le contexte et les interactions de (pouvoirs sur) l'agir. Nous sommes loin de la perspective d'un Sujet autonome, autoengendré et autarcique tel que certaines définitions (ou usages) de la compétence peuvent le suggérer. L'ouverture donnée aux situations, incluant leurs dimensions normatives et techniques, mais également aux différentes formes de l'agir, permet de se rapprocher de la définition de la notion de capacité habilitante développée par exemple en philosophie critique chez Feraresse (Feraresse, 2009).

Cette ouverture étant acquise comme condition de possibilité à une perspective compréhensive et interprétative de l'action, la mobilisation de la notion de jugement réfléchissant devient d'autant plus critique qu'il représente un enjeu pour les soignants et pour les enseignants. Cette notion que Kant reprend au départ de celles de phronesis (grec) et de prudentia (latin) considère cette chose étrange (relativement à la question esthétique chez Kant) : la liberté de poser un jugement universel face à une situation particulière mais sans disposer de catégories universelles. Si le jugement réfléchissant kantien est ainsi vu comme un intermédiaire entre la raison théorique et la raison pratique, nous l'inscrivons pour notre part dans une dimension qui excède cette médiation. À savoir dans une révision pragmatique, inscrite dans les perspectives des théories de l'action, qui dépasse le champ de la seule rationalité. Dans Passion et connaissance, Castoriadis (1997, p. 123) a repris la notion de jugement réfléchissant de Kant, c'est-à-dire le fait que la situation particulière fait émerger une loi (on prétend ainsi dépasser l'expérience empirique individuelle), dans ce qu'il nomme la concrétude particulière, concept qu'il mobilise dans des champs d'action précis, notamment en 
médecine, dans l'enseignement et en politique. En effet, le soignant face à chaque décision (hospitalisation, chirurgie, programme de vaccination, information, etc.) fait de la phronesis, concept mobilisé depuis Hippocrate. Il y a une forme d'angoisse à penser que le médecin ou tout soignant pourrait ne pas se rendre compte de l'incertitude inhérente à la situation particulière et singulière.

Cependant, seule une perspective épistémologique radicalement orientée sur l'action permet d'intégrer en formation cette dimension de liberté et d'incertitude, mais également réflexive et normative de l'agir - particulièrement prégnante dans le cas de l'agir ensemble -, propre à la phronesis. Par ailleurs, si les réflexions sur les pratiques éducatives se sont considérablement développées dans le champ des sciences de l'éducation, elles l'ont fait dans une forte autonomie vis-à-vis des discussions propres aux théories de l'action (Baudouin, 2001, p.12). C'est ainsi que nous ouvrons notre conclusion sur la nécessité de débats, critiques, autour des théories de l'action en éducation dans le secteur de la santé.

Plus spécifiquement, notre choix épistémologique au regard du pluralisme offert par les théories de l'action, est celui de la créativité de l'agir de Hans Joas (2001) qu'il n'est possible d'introduire, ici, que par cette ouverture critique. Nous souhaitons, en effet, insister sur le prolongement du débat au sein même des théories de l'action, qui, historiquement, ont été fondées sur des typologies « de l'action rationnelle, ou encore de l'action téléologique, héritée de la pensée économique du XIXème siècle, qui définit un sujet agissant en fonction d'un but clairement identifié et aménageant une situation et des moyens permettant la réalisation de son projet» (Baudouin, 2001). Ainsi, «rendre compte de manière adéquate d'une action, c'est restituer sa finalité et la cohérence des moyens mis en œuvre "(p.15). Notre "agir-adéquatement-en-santé " (figure 1) s'émancipe d'une telle vision et nécessite un approfondissement de la notion d'intentionnalité, considérant le modèle téléologique, explicatif, comme insuffisant pour désigner la totalité de ce qui importe dans la compréhension de l'action (p.15-16).

Nous sommes maintenant rendus aux limites d'une méthode ou d'un outil, qui, s'il peut prétendre à être condition de possibilité d'une perspective épistémologique et éthique, n'en reste pas moins un outil, un cadre, une méthode, une théorie...dont seule la pratique en déterminera la finalité réelle.

Celle-ci entend l'acquisition d'une sagesse pratique (phronesis) participant au devenir médecin (devenir soignant) (Lefève, 2012) dont rend compte la notion de compétence qui se développe nécessairement dans la durée (longue) et dont le principe de réalité est celui de l'espace-temps. C'est autour de telles responsabilités, celles du choix des « espace-temps » que les méthodes offrent ou n'offrent pas, que l'on peut questionner l'éthique des curriculums de formation en santé. Si depuis Montaigne, nous aimons à redire "J'aime mieux forger mon âme que la meubler ", n'est-il pas envisageable de considérer le concept de compétence comme une opportunité de réalisation pratique d'un tel adage, avec, comme condition de possibilité, la complexité de Morin, permettant d'entrevoir ce qu'on pourrait nommer une épistémologie de la reliance?

Le point commun entre toutes ces personnes, c'est une originalité, une subversion absolument pas artificielle, dans le sens où ce ne sont pas des personnes qui ont souhaité être subversives. Je pense qu'à partir du moment où on a une démarche qui est hors-cadre, hors-norme, on touche aux marges, et on peut être qualifiée à ce titre de sorcière, ou se sentir soi-même sorcière (...). Dans un monde encore trop binaire, la sorcière sait lire entre les lignes, réconcilier, tisser des liens et créer des continuités ... (du Chéné, 2019, p.179). 
Le concept de Reliance, fil conducteur de l'œuvre de Morin (et notion fondatrice pour nous guider dans les limites de notre travail méthodologique) nous amène à conclure ce texte en saluant avec un profond respect Morin en tant que sorcier contemporain que "le mythe moderne du progrès " pour paraphraser le titre de l'ouvrage de Jacques Bouveresse (Bouveresse, 2017) ne permet pas encore suffisamment d'en voir la profondeur et la nécessité.

L'une des variantes de l'erreur de Descartes est de ne pas voir que l'esprit humain est incorporé dans un organisme biologique complexe, mais unique en son genre, fini et fragile; elle empêche donc de voir la tragédie que représente la prise de conscience de cette fragilité, cette finitude et cette unicité. Et lorsque les êtres humains sont incapables d'apercevoir la tragédie fondamentale de l'existence consciente, ils sont moins enclins à chercher à l'adoucir, et peuvent, de ce fait, avoir moins de respect pour la valeur de la vie; (Damasio, 2006, p. 339).

\section{BIBLIOGRAPHIE}

Arora, S., Ashrafian, H., Davis, R., Athanasiou, T., Darzi, A. \& Sevdalis, N. (2010). Emotional intelligence in medicine: a systematic review through the context of the ACGME competencies. Medical Education, 44(8):749-764.

Baudouin, J-M. \& Friedrich, J. (Eds). (2001). Théories de l'action et Education. Louvain-la-Neuve : De Boeck supérieur.

Berthoz, A. Petit, JL. (2006). Phénoménologie et physiologie de l'action. Paris : Odile Jacob.

Bouveresse J. (2017). Le mythe moderne du progrès. Marseille : Editions Agone.

Bozalek, V. \& Zembylas, M. (2017). Diffraction or reflection? Sketching the contours of two methodologies in educational research. International Journal of Qualitative Studies in Education, 30(2): 111-127.

Cassirer, E. (1972). La philosophie des formes symboliques, Tome I (Le langage) (C. Fronty, Trans.). Paris : Les éditions de Minuit (Original work published 1923).

Reed, S. K. (1999). Cognition: théories et applications (T. Blischarski \& P. Casenave-Tapie, Trans.). Bruxelles: De Boeck Université. (Original work published 1996).

Castoriadis, C. (1997). Fait et à faire. Les carrefours du labyrinthe V. Paris : Seuil.

Coutellec, L. (2015). La science au pluriel. Essai d'épistémologie pour des sciences impliquées. Versailles : Quae.

Damasio, A. (2006). L'erreur de Descartes. Paris : Odile Jacob.

De Funès, J. (2019). Développement (im)personnel. Le succès d'une imposture. Paris : Editions de l'Observatoire/Humensis.

De Ketele., J-M. \& Delory, C. (2000). Quel héritage pédagogique pour le nouveau millénaire?

Pédagogies Forum, 35, 9-16. 
Deledalle, G. (2018). La pédagogie de John Dewey. In John. Dewey, Démocratie et éducation : Suivi de Expérience et Éducation (pp. 7-44). Paris : Presses Universitaires de France.

Dewey, J. (2015). L'art comme expérience (J-P. Cometti, Ch. Domino, F. Gaspari, C. Mari, N. Murzilli, C. Pichevin, J. Piwnica et GA. Tiberghien, Trans.). Paris : Folio essais. (Original work published 1923).

Dewey, J. (2008). La théorie de la valuation (A. Bidet, Trans.). Tracés. Revue de Sciences humaines, 15, 217-228 doi.org/10.4000/traces.833. (Original work published : Dewey, J. (1939). Theory of valuation, International Encyclopedia of Unified Science, 2(4):1-67.

du Chéné., C. (2019). Les sorcières. Paris : Michel Lafon / Editions France Culture.

Doidge, N. (2015). Guérir grâce à la neuroplasticité. Paris : Belfond.

Durand, D. (2010). La systémique. Que sais-je ? Paris : Presses universitaires de France

Eoyang, G.H. \& Menning, S. (2019). Wicked Problems in Health Professions Education: Adaptative Action in Action. MedEdPublish, 1-11. doi.org/10.15694/mep.2019.000226.1

Ferrarese, E. (2009). Vivre à la merci. Le care et les trois figures de la vulnérabilité dans les théories politiques contemporaines. Multitudes, 2(37-38), 132-141.

Folscheid, D. (1996). La médecine comme praxis : un impératif éthique fondamental. Laval Théologique et Philosophique, 52:499-509.

Foucart, J. (2013). Pragmatisme et transaction. La perspective de John Dewey. Pensée plurielle, 33-34(2), 73-84.

Joas, H. (2001). La créativité de l'agir. In J-M Baudouin \& J Friedrich (Dir.). Théories de l'action et Education. (pp. 27-43). Louvain-la-Neuve : De Boeck supérieur.

Jorro A. \& De Ketele J-M. (Eds) (2011). La professionnalité émergente : quelle reconnaissance ? Louvainla-Neuve : De Boeck.

Jouquan, J., Parent, F., Mathé, F., Burucoa, B. (2020, sous presse). Penser le développement des soins palliatifs en termes de compétences. In R Le Berre (Dir.). Manuel de soins palliatifs, Paris : Dunod

Jouquan, J. Parent, F. (2013). «Les enjeux épistémologiques et méthodologiques de l'évaluation des pratiques professionnelles en santé » in : Parent, F. Jouquan, J. Penser la formation des professionnels de la santé. Une perspective intégrative. Louvain-la-Neuve : De Boeck.

Kant, E (1985). CEuvres philosophiques, tome 2, (F. Alquié, AJ.-L. Delamarre, V. Delbos, J. Ferrari, L. Ferry, F. de Gandt, P. Jalabert, J-R. Ladmiral, M. de Launay, B. Lortholary, F. Marty, J. et O. Masson, A. Philonenko, A. Renaut, J. Rivelaygue, J-M. Vaysse, H. Wismann \& S. Zac. Trad.) Paris : Gallimard, Bibliothèque de la Pléiade (Publication originale 1790).

Lefève, C. (2012). Devenir médecin. Cinéma, formation et soin. Paris : Presses Universitaires de France. Leibniz, Lettre à Lambert van Velthuysen, 5 mai 1671, A, II, I, p. 98 ; Elementa juris naturalis, A, VI, I.

Legendre, R. (2006). Dictionnaire actuel de l'éducation. Montréal : Guérin.

Le Moigne, J-L. (2012). Les épistémologies constructivistes (4e éd.). Paris : Presses Universitaires de France.

Monet, E. (2007). La théorie des « capabilités » d'Amartya Sen face au problème du relativisme. Tracés. Revue de Sciences humaines, 12,103-120. doi.org/10.4000/traces.211. 
Morin, E. (2006). Les sept savoirs nécessaires. Revue du MAUSS, 2006/2(28), 59-69.

Morin, E. (2004). La méthode. VI. Éthique, Paris : Le Seuil.

Morin, E. (1997). Réforme de pensée, transdisciplinarité, réforme de l'Université. Communication au Congrès International "Quelle Université pour demain ? Vers une évolution transdisciplinaire de l'Université " (Locarno, Suisse, 30 avril - 2 mai 1997). In Motivation, 1997,24.

Morin, E. (1990). Science avec conscience. Paris : Le Seuil.

Parent, F., Jouquan, J. (2019). Entrée « développement personnel continu ». In C. Boelen, M. Cauli, J. Ladner. Dictionnaire francophone de responsabilité sociale en santé (pp. 170-175). Mont SaintAignan : Presses Universitaires de Rouen et du Havre,

Parent, F., Jouquan J. (2015). Comment élaborer et analyser un référentiel de compétences en santé dans une perspective intégrative. Une clarification conceptuelle et méthodologique critique de l'approche par compétences. Louvain-la-Neuve : De Boeck.

Parent, F., Jouquan, J. (2014). Taxonomies et typologies : pas synonymes mais complémentaires. Santé publique, 26(1):85-6.

Parent, F, Jouquan J. (2014). Taxonomies et typologies : quelques clarifications complémentaires. Santé publique, 26(2) :227-228.

Parent, F., Jouquan, J., Kerkhove, L., Jaffrelot, M., De Ketele, J-M. (2012). Intégration du concept d'intelligence émotionnelle à la logique de l'approche par compétences dans les curriculums de formation en santé. Pédagogie Médicale, 183-201.

Poumay, M., Tardif, J. (2017). Organiser la formation à partir des compétences - Un pari gagnant pour l'apprentissage dans le supérieur. De Boeck Supérieur.

Roger, A. (1997). Court traité du paysage. Paris : Folio essais.

Schurmans, M-N. (2006). Expliquer, interpréter, comprendre. Le paysage épistémologique des sciences sociales. Carnets des sciences de l'éducation. Genève, Publications de l'Université de Genève.

Steiner, P. (2008). Sciences cognitives, tournant pragmatique et horizons pragmatistes. Tracés. Revue de Sciences humaines,15,85-105. doi.org/10.4000/traces.663

van der Kolk, B. (2018). Le corps n'oublie rien. Le cerveau, l'esprit et le corps dans la guérison du traumatisme. Paris : Albin Michel.

van Grunsven, J. (2015). Bringing Life in View. An Enactive Approach to Moral Perception. Dissertation: The New School for Social Research, NY.

Varela, F. (1988). Invitation aux sciences cognitives. Paris : Seuil.

Venturini, P. (2012). Action, activité, « agir » conjoints en didactique : discussion théorique.

Éducation et didactique, 6(1), 127-136

\section{NOTES}

1. Nous faisons référence ici aux travaux de Venturini définissant au regard d'une démarche de didactique professionnelle les notions d'expérience, d'activité et d'action rejoignant ainsi des travaux publiés antérieurement sur l'approche par compétences intégrée.

2. En référence à l'éthique du genre humain.

3. Cette figure est adaptée à partir de travaux du réseau thématique «Éthique des curriculums en santé » de la Société internationale francophone d'éducation médicale (SIFEM). 
4. Nous entendons le concept de reliance tel qu'il a été approfondi par Edgar Morin dans le cadre de développements successifs, en lien avec son approche de la complexité. Il indique ainsi que « [1] notion de reliance [...] comble un vide conceptuel en donnant une nature substantive à ce qui n'était conçu qu'adjectivement, et en donnant un caractère actif à ce substantif. "Relié" est passif, "reliant" est participant, "reliance" est activant ".

5. Cassirer, E., La philosophie des formes symboliques, pp. 224-225: «Si l'on envisage maintenant l'ensemble des différentes formes verbales et si l'on songe que la plupart de ces formes peuvent se réunir pour constituer de nouvelles unités complexes, on se rend compte que, si le langage peut inventer de telles formes, c'est parce qu'il ne conçoit pas l'opposition de l'être objectif et de l'être subjectif comme l'opposition abstraite et figée de deux domaines qui s'excluraient réciproquement et qu'au contraire il pense cette opposition de manière très complexe et qu'il voit en elle une opposition dynamique et médiatisée $\{. .$.$\} Le langage ne présente pas deux sphères$ en elles-mêmes, il montre comment elles interfèrent et comment elles se déterminent réciproquement ; il ouvre, pour ainsi dire, un domaine intermédiaire à l'intérieur duquel les formes d'existence se rapportent aux formes de l'action, et vice versa, et dans lequel elles se fondent en une seule unité expressive. "

6. Les critères ayant permis d'orienter cette structuration sont explicités dans l'ouvrage : "Comment élaborer et analyser un référentiel de compétences en santé » et, plus particulièrement, dans l'encadré intitulé «Des nouveaux domaines taxonomiques à défricher dans le cadre de l'approche par compétences : une réflexion autour du domaine imaginatif » (Parent \& Jouquan, 2015, p. 168).

\section{RÉSUMÉS}

En hommage à Edgar Morin, nous centrons la présente contribution sur le débat de la complexité en éducation, dans un contexte spécifique celui des planifications curriculaires et des programmes de formation en santé, et sur un objet particulier, celui des taxonomies en éducation. Au départ, nous formulons un triple postulat : épistémologique, celui d'un glissement de l'objet d'enseignement et d'apprentissage du savoir vers la pratique dans une perspective constructiviste (Le Moigne, 2012) et pragmatiste (Steiner, 2008) ; anthropologique, celui d'une ouverture à la pluralité des savoirs (Coutellec, 2015 ; Schurmans, 2006) permettant de rencontrer l'être humain dans toute sa complexité ; éthique, celui d'une interprétation non téléologique de l'intentionnalité de l'agir humain en référence aux travaux de Hans Joas (Joas, 2001). Le concept de «l'agir-adéquatement-en-santé » présente ce triple postulat de départ et constitue l'objet de la taxonomie de l'approche par compétences intégrée au regard des quatre critères de la systémique de Durand (2010) : la globalité, l'organisation, l'interaction et la complexité. Outre les ouvertures réflexives émises lors de la présentation de la taxonomie (comme celles liées à la reliance, à la relecture husserlienne du sujet transcendantal), la discussion finale en soulève d'autres comme la nécessité d'approfondir et de prolonger la réflexion de Kant en faisant appel, d'une part aux théories de l'action, et d'autre part à la théorie de la créativité de l'agir développée par Hans Joas (2001).

As a tribute to Edgar Morin, we focus this paper on debating about complexity in education, in the specific context of curriculum planning and health care education programs, about a particular object, namely taxonomies in education. As a starting point we assume a threefold 
postulate : an epistemological one, which puts forward the need of a shift from knowledge toward practice, in order to delineate teaching and learning object, according to a constructivist (Le Moigne, 2012) and pragmatist (Steiner, 2008) perspective ; an anthropological one, which promotes openness to knowledge plurality (Coutellec, 2015; Schurmans, 2006), in order to meet the complexity of human being ; and, finally, an ethical one, aiming a non-teleological interpretation of the intentionality of human action, with reference to Hans Joas' work (Joas, 2001). The concept of "acting-adequately-in-health" is consistent with theses starting premises and constitutes the object of the taxonomy associated with the so-called integrated competency-based model, with regard to the four criteria linked to the systemic approach of Durand (2010): globality, organization, interaction and complexity. In addition to a reflexive presentation of taxonomy (relating to the concept of connectedness or to a reinterpretation of the Husserlian transcendental subject), the final discussion raises others concerns such as the need to deepen and extend Kant's reflection by appealing both to action theories and to creative action theory, as developed by Hans Joas (2001).

\section{INDEX}

Mots-clés : épistémologie, reliance, taxonomie, éthique, complexité

Keywords : epistemology, connectedness, taxonomy, ethics, complexity

\section{AUTEURS}

\section{FLORENCE PARENT}

Université libre de Bruxelles (Belgique), Faculté de médecine, Bruxelles (Belgique)

\section{JEAN-MARIE DE KETELE}

Université catholique de Louvain, Louvain-la-Neuve (Belgique), Chaire UNESCO en sciences de l'éducation, Dakar (Sénégal), Institut Catholique de Paris, Paris (France)

\section{FABIENNE GOOSET}

Université libre de Bruxelles (Belgique), Ecole de Santé Publique, Collaboratrice scientifique au Centre de recherche « Economie de la santé - Gestion des institutions de soins - Sciences infirmières ", Bruxelles (Belgique)

\section{MANOÉ REYNAERTS}

Haute école libre de Bruxelles - Ilya Prigogine, Bruxelles (Belgique) 